\title{
Synchronous measurement of tribocharge and force at the footpads of freely moving animals
}

\author{
Yi SONG ${ }^{1,2}$, Zhouyi WANG ${ }^{1,3}$, Jun ZHOU ${ }^{3}$, Yang LI $^{3}$, Zhendong DAI ${ }^{1,3, *}$ \\ ${ }^{1}$ Institute of Bio-inspired Structure and Surface Engineering, Nanjing University of Aeronautics and Astronautics, Nanjing 210016, China \\ ${ }^{2}$ College of Mechanical and Electrical Engineering, Nanjing University of Aeronautics and Astronautics, Nanjing 210016, China \\ ${ }^{3}$ College of Astronautics, Nanjing University of Aeronautics and Astronautics, Nanjing 210016, China \\ Received: 08 January 2017 / Revised: 13 April 2017 / Accepted: 02 May 2017 \\ C The author(s) 2017. This article is published with open access at Springerlink.com
}

\begin{abstract}
Hypothesis on electrostatic attraction mechanisms involving the hairy adhesion of climbing animals has been a matter of controversy for several years. The detection of tribocharge and forces at attachment organs of animals is a practical method of clarifying the dispute with respect to electrostatic attraction in the attachment of animals. Nonetheless, the tribo-electrification is rarely examined in the contact-adhesion of animals (especially in their free and autonomous attachment) due to the lack of available devices. Therefore, the present study involves establishing a method and an apparatus that enables synchronous detection of tribocharge and contact forces to study tribo-electrification in the free locomotion of geckos. A type of a combined sensor unit that consists of a three-dimensional force transducer and a capacitor-based charge probe is used to measure contact forces and tribocharge with a magnitude corresponding to several nano-Coulombs at a footpad of geckos when they climb vertically upward on an acrylic oligomer substrate. The experimental results indicate that tribocharge at the footpads of geckos is related to contact forces and contact areas. The measured charge allows the expectation of an exact attraction with magnitude corresponding to dozens of newtons per square meter and provides a probability of examining tribo-electrification in animal attachment from a macro level.
\end{abstract}

Keywords: tribocharge; forces; synchronous measurement; animal; free locomotion

\section{Introduction}

An understanding of adhesion mechanisms in the hierarchical attachment organs of animals is of immense scientific significance and engineering potential [1-5]. However, the adhesion mechanism of animals' hairy attachment organs continues to constitute a controversial matter. Although extant studies over several years confirmed that van der Waals interaction $[6,7]$ and capillary forces $[8,9]$ play dominant roles in setae adhesion, electrostatic attraction is increasingly invoked $[10,11]$ to interpret the adhesion of hairy systems $[9,10]$. It is easy to positively tribo-electrify hair [12, 13], and thus animals' hairy attachment organs are expected to become charged and subsequently generate electrostatic forces [10,14] as long as they interact with the environment to obtain forces to drive their locomotion. Unfortunately, there is a paucity of definite evidence to confirm the electrostatic attraction hypothesis in animal dry adhesion (especially involving free movement) due to the lack of feasible equipment.

Measurement of the tribocharge (the charge resulting from contact and friction) and forces at the feetsubstrate interfaces is a practical and effective method to investigate tribo-electrification and electrostatic interaction in animal attachments. Additionally, it is important to synchronously implement the measurements to reduce the error caused by charge dissipation and/or neutralization. Although facilities for measuring force $[15,16]$ and charge $[17-21]$ are

* Corresponding author: Zhendong DAI, E-mail: zddai@nuaa.edu.cn 
well developed, the synchronous measurement of both remains a challenge. Chiou et al. measured tribovoltage between two metals through a voltmeter and obtained contact forces by using a lever system [22]. Budakian et al. developed a type of rotary vane apparatus to study tribo-electrification between a sphere and a plate and obtained corresponding forces through a deformed cantilever [23]. Furthermore, a previous study used atomic force microscopes to measure tribocharge and forces between micro/nano objects [24]. However, to the best of the authors' knowledge, extant studies to date have not applied these techniques with respect to moving animals.

Based on electrostatic induction, Izadi et al. [14] measured tribocharge and shearing forces at the footpads of geckos that were compelled to slide on polytetrafluoroethylene AF (PTFE AF) film and polydimethylsiloxane (PDMS) film. However, in their experiments, animals were only compelled to interact with a substrate instead of attaching autonomously, and this, for one thing, may cause a deviation in the results, and for another, limited the practicability of their apparatus.

Therefore, the present study involved developing a technique and an apparatus with the aim of examining trio-electrification in the attachment of moving animals. The apparatus allowed the synchronous measurement of tribocharge and forces at the footpads of geckos when they climbed vertically upwards. Electrostatic attraction and its possible contribution were also preliminarily discussed based on the experimental outcomes. The successful synchronous measurement provided a method to conduct an in-depth investigation of tribo-electrification in animal attachment.

\section{Materials and methods}

\subsection{Animals}

Four healthy Gekko geckos (Linnaeus) with an average mass of $86.8 \pm 7.2 \mathrm{~g}$ (mean \pm s.d.) that originally inhabited Guangxi province (China) were employed in the experiments. They were raised in a room with a supply of live crickets and under simulated natural conditions including decorations involving rockworks, a water pool, a lighting system (with a cycle of $12 \mathrm{~h}$ of light and $12 \mathrm{~h}$ of darkness), air conditioning $\left(23 \pm 2{ }^{\circ} \mathrm{C}\right)$, and a humidity regulator $(60 \%-70 \%)$, before and after the experiments.

\subsection{Techniques and apparatus}

In summary, the system comprised of five subparts and included sensor units, a rotatable aisle, an image recorder, a data acquisition (DAQ) module, and a computer (PC) as shown in Fig. 1.

Specifically, a sensor unit (as shown in Fig. 1, a) that detected both the tribocharge and forces was embedded in a rotatable aisle (as shown in Fig. 1, b)

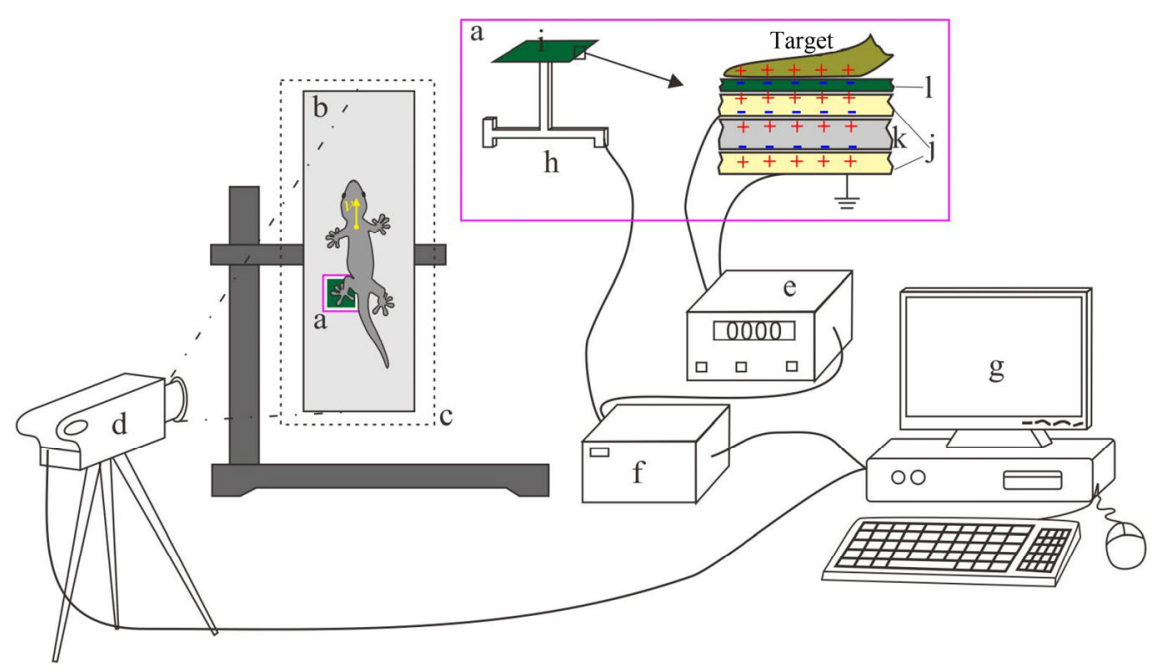

Fig. 1 Testing techniques: a-a sensor unit; $b$-a rotatable metal aisle; $c-a$ metal cage; $d-a$ high-speed camera; e - a digital coulometer; $\mathrm{f}-\mathrm{a}$ NI DAQ module; $\mathrm{g}$ - a PC; $\mathrm{h}$-a 3d force sensor; $\mathrm{i}$-a charge probe; $\mathrm{j}$-copper foils; $\mathrm{k}$-an epoxy glass fiber plate; 1 -an insulator film. 
that was well-grounded to guide possible charge at the animals' feet into the earth prior to contact with the probes. The packaged aisle was fully protected by a metal cage (Fig. 1, c) to prevent environmental electromagnetic noises from affecting the test. A high-speed camera (Fig. 1, d) (i-speed 3, Olympus, Japan) was placed in front of the aisle with its lens perpendicular to the aisle to record the animal-substrate interaction throughout the experiment. The charge signals were converted through a digital coulometer (ES111, ESDEMC Technology, Rolla, Missouri, USA, Fig. 1, e) prior to collecting the same with a DAQ system (National Instruments, TX, USA, Fig. 1, f). In contrast, the force signals were collected straight through the DAQ module and were subsequently transmitted to the PC (Fig. 1, g). The video recording and storing were directly completed on the PC.

In hardware terms, the probe simultaneously functioned as a charge sensor and force carrier to ensure synchronous detections of force and charge signals. In software terms, a trigger signal was generated and subsequently divided into three to trigger the subsystems such that the tribocharge, contact forces, and videos were conjointly recorded.

The sensor unit consisted of a three-dimensional force sensor with scale range of $1.5 \mathrm{~N}$ and a resolution of $2 \mathrm{mN}$ (as shown in Fig. 1, h) and a capacitor-based charge probe (as shown in Fig. 1, i). In order to accurately measure charge, it is necessary for the capacity of the probe to be sufficiently small in conjunction with a sufficiently large resistance [25], and this results in a large mass of the probe that can dramatically weaken the dynamical performance of the force sensor [26]. As a compromise, a probe was fabricated by laminating copper foils $(25 \mu \mathrm{m}$ in average thickness, Fig. 1, j) on an industrial FR-4 epoxy glass fiber plate (as shown in Fig. 1, k) with a size corresponding to $30 \mathrm{~mm} \times 30 \mathrm{~mm} \times 0.8 \mathrm{~mm}$, a resistance corresponding to $10^{14} \Omega$, a capacity corresponding to $70.2 \mathrm{pF}$ (smaller than one thousandth of that of the coulometer), and a mass corresponding to $2.06 \mathrm{~g}$. Additionally, a thin acrylic oligomer film (as shown in Fig. 1, 1) was coated at the top surface of the probe as a substrate to interact with the animals' feet.

Animals' feet move unpredictably, and thus it is difficult to track the object accurately in real time. Hence, the charge at the substrate that is equal to albeit with an opposite sign to the charge at the objects $[12,27]$ was alternatively obtained.

The system was calibrated before and after the experiments. The force sensor was calibrated with a dead weight (Fig. 2(a)) [16] while the coulometer was calibrated based on the user's manual [28] (Fig. 2(b)). If the total capacity of the system corresponded to $C$, the system obtains a total charge of $Q=C V_{D}$ when being charged by a voltage $V_{\mathrm{D}}$. Thus, it is possible to calibrate the coulometer by comparing the measured charge and the theoretical charge (as shown in Table 1).

\subsection{Experiments and data process}

Prior to the animal experiments, the surface profile of the polymer substrate was observed by performing super field depth 3D microscopy (VHX-600E, Keyence, Japan) to estimate its roughness.

The tribocharge and three-dimensional forces at the geckos' feet were synchronously measured when
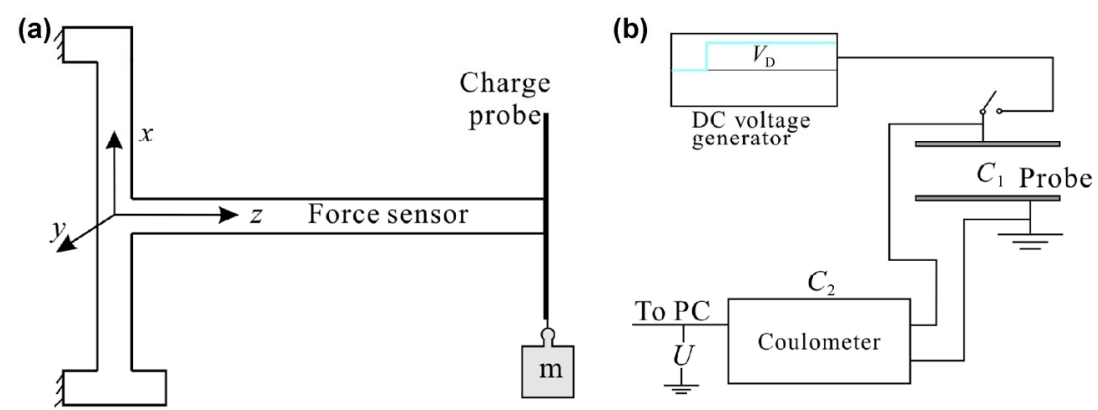

Fig. 2 Calibrations of sensor units. (a) A sketch illustrating the force calibrating method. (b) The calibrating method for the charge testing (adapted from the user's manual) in which $V_{\mathrm{D}}$ denotes supplied voltage and $C_{1}$ and $C_{2}$ denote the capacities of probe and coulometer, respectively. The total capacity and resistance of the system correspond to $100.072 \mathrm{nF}$ and $0.6 \Omega$, respectively, and result in a time constant corresponding to $6.0042 \times 10^{-8} \mathrm{~s}$. 
Table 1 Summary of the performance of the sensors.

\begin{tabular}{|c|c|c|c|c|c|c|}
\hline \multicolumn{2}{|c|}{ Sensor type } & Full scale (FS) & Coupling $(\% \mathrm{FS})$ & Non-linearity $(\% \mathrm{FS})$ & Hysteresis $(\% \mathrm{FS})$ & Resolution \\
\hline \multirow{3}{*}{ Force } & $x$ & $1,500 \mathrm{mN}$ & 1.29 & 0.13 & 0.21 & $2 \mathrm{mN}$ \\
\hline & $y$ & $1,500 \mathrm{mN}$ & 1.74 & 0.14 & 0.11 & $2 \mathrm{mN}$ \\
\hline & $z$ & $1,500 \mathrm{mN}$ & 2.10 & 0.13 & 0.21 & $3 \mathrm{mN}$ \\
\hline \multicolumn{2}{|c|}{ Charge } & $2 \mathrm{nC}$ & - & $<0.01$ & $<0.01$ & $0.1 \mathrm{PC}$ \\
\hline
\end{tabular}

they climbed vertically upward, at a sample rate of $500 \mathrm{~Hz}$ in an isolated room that was under sufficient electromagnetic protection with a temperature corresponding to $22-25{ }^{\circ} \mathrm{C}$ and a humidity of $60 \%-70 \%$. The aisle and probe surfaces were carefully cleaned with alcohol (analytically pure, Nanjing Chemical Reagent Co. Ltd.) before every trial. The complete test system and the animals were electrically neutralized with an ionizing air gun prior to the experiment to diminish the influence of the residual charge on the results. Additionally, anti-static clothing and gloves were utilized throughout the experiment.

Trials in which geckos moved discontinuously or exhibited unstable attachment were eliminated to diminish the uncertainty of the results.

The force signals and charge signals were processed in MATLAB (The Mathworks, Inc, Mass, USA) by using a method used in a previous study [16]. The recorded videos were decomposed into images such that the nominal contact areas $A$ were acquired through image processing.

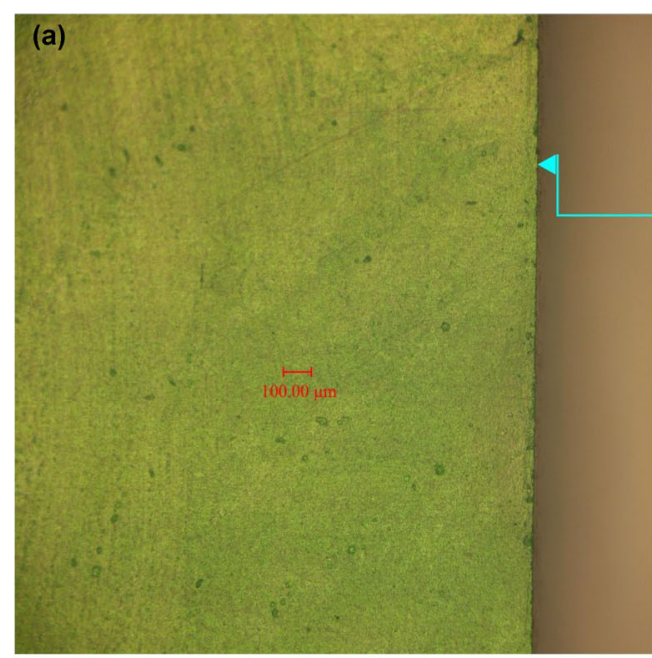

\section{Results}

A top view of the charge probe was shown in Fig. 3(a) whereas a lateral view was shown in Fig. 3(b). The substrate corresponded to an insulator film with an average thickness of $12.4 \mu \mathrm{m}$. Due to surficial defects, such as scratches, the film surface was not very smooth and was full of micro pits and asperities (Figs. 3(a) and $3(\mathrm{c}))$. A calculation of the exact surface profiles led to obtaining a root mean square roughness $(\mathrm{Rq})$ of $0.69 \mu \mathrm{m}$ and a mean roughness ( $\mathrm{Ra}$ ) of $0.48 \mu \mathrm{m}$ for the insulator substrate.

When a gecko treaded on a sensor unit (Fig. 4(a)) embedded in the aisle, its foot interacted with the polymer substrate to obtain forces that caused its locomotion, and this resulted in synchronous tribocharge and forces at the foot-polymer interfaces. The charge was generated at the moment when the foot of the gecko came into contact with the substrate, and it increased steadily before it reached a maximum at the moment of separation (Fig. 4(b)). Additionally,

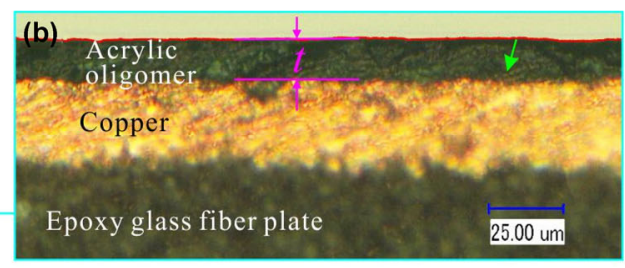

(c)

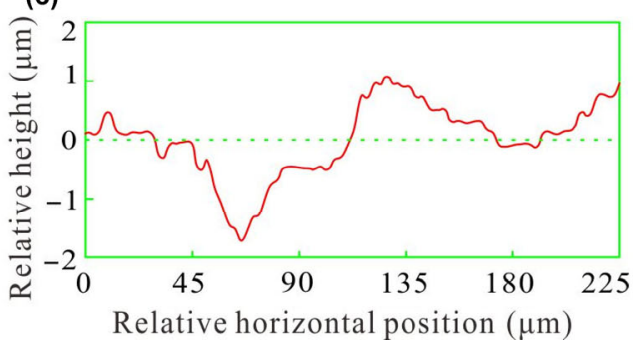

Fig. 3 The surface profile of the substrate. (a) A top view of the probe surface. (b) A lateral view of the section of the probe. (c) A typical surficial profile of the probe surface. 
the peaks of frictional adhesion at the geckos' hind footpads $\left(F_{S}\right)$ that resulted from the forces in fore-aft direction $\left(F_{y}\right)$ and lateral direction $\left(F_{x}\right)$ increased with increases in the nominal contact areas and exhibited a relationship corresponding to $F_{\mathrm{S}}=1.95 \times 10^{-3} \mathrm{~A}$ $\left(R^{2}=0.71, n=24\right)$. In contrast, the results indicated that the normal force peaks $\left(F_{z}\right)$ are irrelevant with respect to the contact areas (Fig. 4(c)). Interestingly, when the geckos' seta arrays interacted with the polymeric films on a vertical wall, the geckos' hairy foot pads generally exhibited a positive charge with a magnitude ranging from a few tenths of a nanoCoulomb to a few nano-Coulombs. Furthermore, the charge increased with increases in the contact areas ( $R^{2}=0.79, n=24$ ) (Fig. 4(d)). As shown in Fig. 4(e), the contact charge was evidently related to the contact forces. An increase in the frictional adhesion led to a significant increase in the contact charge $\left(R^{2}=0.86\right.$, $n=24)$

\section{Discussion}

As shown in Fig. 3, the substrate that interacted with geckos' footpads was not as smooth as mirrors and instead possessed a roughness of $\mathrm{Rq} 0.67$ that was caused by surficial defects such as scratches. It should be noted the roughness of the substrate significantly exceeded the roughness span ( $\mathrm{Rq} 0.1-0.3$ ) in which the geckos could hardly adhere to the wall [29]. Thus, it was concluded that geckos could sufficiently contact the substrate without requiring any macroscopic sliding and generated reliable forces. When the geckos climbed vertically upward, their bodies moved sinusoidally (as shown in Fig. 4(a) insert), and this resulted in a wave-like locomotion [30] with a velocity of $0.5 \pm$ $0.1 \mathrm{~m} / \mathrm{s}(n=24)$, and this was in agreement with observations in previous studies [31, 32].

When a gecko interacted with the insulator substrate through its feet to obtain forces that drove or retarded its locomotion, the tribocharge was generated at the moment when the hairy footpads were in contact with the substrate and began increasing before it plateaued at the moment of contact separation (Fig. 4(b)). This was in agreement with the property of tribo-electrification. Specifically, the charge increased steadily even when the dominant forces $\left(F_{y}\right)$ already decreased (Fig. 4(b)), and this indicated that this part of the charge resulted from frictional contact. Moreover,
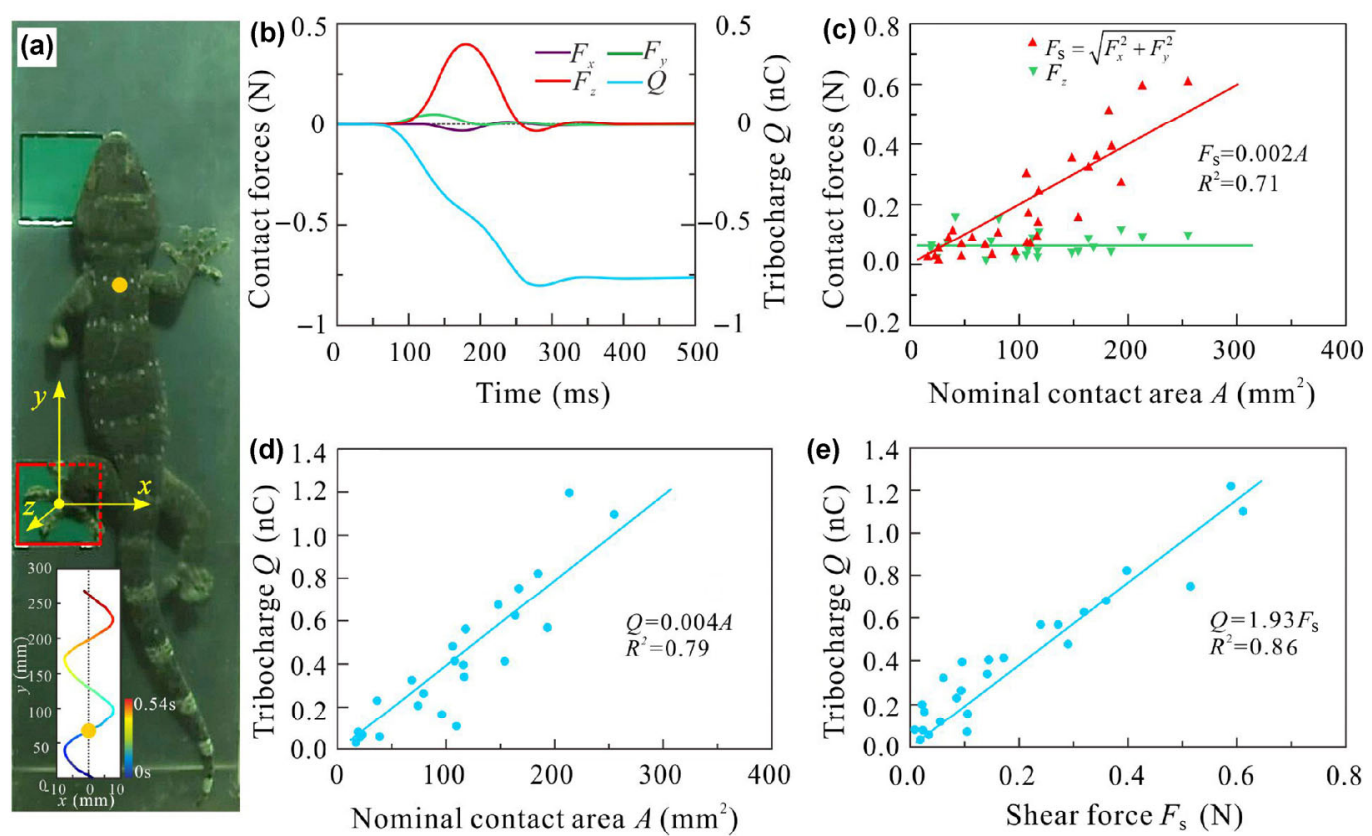

Fig. 4 Experiment results. (a) An illustration of the interaction between a gecko's foot and the insulator film; insert, the trace of the middle point of the gecko's front shoulders. (b) Typical test results. (c) The relationship between nominal contact areas and forces. (d) The relationship between a contact area and tribocharge. (e) The relationship between tribocharge and contact forces. The data are obtained from the left hind feet, and therefore the normal forces are positive. 
the geckos' attachment completely constituted a type of frictional contact since their foot hairs could only adhere to a target following relatively micro slips [7]. Therefore, it was concluded that the measured tribocharge mainly resulted from the friction between geckos' foot hairs and the acrylic oligomer film. Extant studies on other materials indicated that the tribocharge accumulation rate strongly depended on the contactseparation speed [12], and this may also hold for the tribo-electrification of geckos. It should be noted that the geckos moved with a similar speed in the selected trials, and the influence of contact-separation speed was not revealed here.

Linear regressions shown in Fig. 4(c) illustrate that the frictional adhesion forces $\left(F_{\mathrm{s}}\right)$ are linearly related to the nominal contact areas $A\left(R^{2}=0.71, n=24\right)$ and result in a nominal strength of $0.17 \pm 0.07 \mathrm{~N} / \mathrm{cm}^{2}$. Conversely, the normal forces $F_{z}$ were irrelevant to the nominal contact areas. The geckos' toes constitute a type of soft and anisotropic structure that is proficient at generating adhesion and at bearing tensile stresses. However, they are incapable of sustaining compressive stresses due to the small and irregular normal forces at the soft toes of the hind limbs of the geckos. Furthermore, this indicated that a large normal force was not indispensable for adhesive friction in geckos.

The study involved measuring the charge at the substrate instead of that at the moving target, and thus the negative charge at the insulator film suggested that an equal albeit positive charge existed at the geckos' footpads. This was in agreement with the fact that human hairs are always positively charged when they are rubbed with polymers $[13,33]$ because the geckos' foot hair possessed components similar to human hairs [34]. Despite the relatively low correlation $\left(R^{2}=0.79, n=24\right)$, the tribocharge appeared to increase with increases in the contact areas in the experiment (Fig. 4(d)). This was consistent with the results reported in materials used in previous studies [35, 36]. Generally, large contact areas allow increased setae-polymer interaction that contributes to both tribocharge and forces.

Interestingly, the study findings indicated that the tribocharge significantly increased with an increase in the frictional adhesion forces $\left(R^{2}=0.86, n=24\right)$ (Fig. 4(e)). Potentially, the large forces enhanced the electrification that occurred at the seta-solid interfaces. Obviously, the tribocharge divided by the contact areas gives a charge density $(\sigma)$ of $4.05 \pm 1.03 \mathrm{pC} / \mathrm{mm}^{2}$ $(n=24)$. However, it should be noted that the charge density is definitely underestimated here for two reasons. For one thing, the measured charge (i.e., the charge induced by the real tribocharge) is generally a little inferior to the actual ones. For another, the nominal contact areas are considerably overnumber the actual ones and probably even larger than quintuple of the same [37]. Interestingly, the comparison between the nominal frictional adhesion $\left(0.19 \mathrm{~N} / \mathrm{cm}^{2}\right)$ here and the actual frictional adhesion corresponding to a gecko's foot on polymers (for example, $2.3 \mathrm{~N} / \mathrm{cm}^{2}$ on PTFE [14]) approximately estimated the actual contact areas as corresponding to about one tenth of the nominal areas. Thus, the actual charge density should significantly exceed $4.05 \mathrm{pC} / \mathrm{mm}^{2}$. Nevertheless, the density of tribocharge that was determined as corresponding to the footpads of freely, vertically, and upward climbing geckos was still smaller than that reported by Izadi et al. [14]. This is because Izadi et al. used different materials (PTFE AF and PDMS) that correspond to the most negative position of the tribo-electric series $[35,36]$ as a substrate. Additionally, the hairy footpads were compelled to slide on the substrate for approximately $10 \mathrm{~mm}$ in their experiment, and the long sliding significantly benefited the tribo-electrification [38]. Moreover, high humidity may also weaken the tribo-electrification in the experiment performed in the present study.

A parallel plate capacitor model was used to approximately estimate the electric field force per unit area that results from the tribo-charge if the boundary effects are overlooked [27] as follows:

$$
F=-\frac{\sigma^{2}}{2 \varepsilon}
$$

where $\varepsilon$ denotes the effective permittivity. The above charge density was substituted into Eq. (1) to obtain a nominal electrostatic attraction strength that approximately corresponded to $1 \mathrm{~N} / \mathrm{m}^{2}$ if the charge dissipation was ignored. Considering the negative deviation of measured charge density, an exact electrostatic attraction with a magnitude of approximately dozens of to hundreds of newtons per square meter would 
be generated if a more precise charge density was obtained.

Nonetheless, the electrostatic attraction is still significantly smaller than that obtained by Izadi et al. by pulling a gecko's foot on a PTFE AF film for a few millimeters (approximately $4.7 \times 10^{4} \mathrm{~N} / \mathrm{m}^{2}$ ) [14]. The disparity indicates that the electrostatic interaction at the setae-acrylic oligomer interface may not function as dominantly as the claim in the previous study when geckos move freely. Given that the function range of electrostatic interaction significantly exceeded that of van der Waals interaction, the electrostatic attraction was ideally expected to function in the next step from a distance in which the van der Waals forces were unable to function to reduce the setae-substrate gap such that the van der Waals force could come into effect. However, the assumption was still indeterminable and will be strictly verified in future experiments.

\section{Conclusions}

Conclusively, the present study involved using a self-developed apparatus to measure the tribocharge at geckos' footpads when they climbed vertically upwards. The successful synchronous measurement provides a method of deeply investigating triboelectrification in animal attachment. Interestingly, the results indicate that the tribocharge is related to the frictional forces and contact areas. The experiments illustrate a nominal charge density of $4.05 \mathrm{pC} / \mathrm{mm}^{2}$ that generates a nominal electrostatic attraction corresponding to $1 \mathrm{~N} / \mathrm{m}^{2}$. An electrostatic attraction with a strength corresponding to approximately 25$100 \mathrm{~N} / \mathrm{m}^{2}$ is expected to be obtained given that the actual contact areas as opposed to the nominal areas are applied for the calculation. However, the electrostatic attraction continues to remain smaller than the van der Waals forces.

There are still a great many of issues unclear additional to this preliminary study on the triboelectrification in animal attachment. As a continuation, the issues including the actual contact areas of freely moving animals, the influence of substrate materials on the charge, and the precise extent to which the tribocharge contributes to the adhesion and friction will be further investigated.

\section{Acknowledgements}

The authors extend their sincere appreciation to Wenbo Wang for raising geckos and aiding in performing the 3D microscopy and to the reviewers for their constructive advices. This study was supported by grants from the National Natural Science Foundation of China (Grants No. 51435008) and funding from the Jiangsu Innovation Program for Graduate Education (Grants No. KYLX16_0328).

All applicable institutional and/or national guidelines for the care and use of animals were followed in the study.

Open Access: The articles published in this journal are distributed under the terms of the Creative Commons Attribution 4.0 International License (http:// creativecommons.org/licenses/by/4.0/), which permits unrestricted use, distribution, and reproduction in any medium, provided you give appropriate credit to the original author(s) and the source, provide a link to the Creative Commons license, and indicate if changes were made.

\section{References}

[1] Persson B N J. On the mechanism of adhesion in biological systems. J Chem Phys 118(16): 7614-7621 (2003)

[2] Gorb S N, Varenberg M, Peressadko A, Tuma J. Biomimetic mushroom-shaped fibrillar adhesive microstructure. J R Soc Interface 4(13): 271-275 (2007)

[3] Qu L T, Dai L M, Stone M, Xia Z H, Wang Z L. Carbon nanotube arrays with strong shear binding-on and easy normal lifting-off. Science 322(5899): 238-242 (2008)

[4] Wang Z Y, Song Y, Dai Z D. Use of opposite frictional forces by animals to increase their attachment reliability during movement. Friction 1(2): 143-149 (2013)

[5] Zhou M, Pesika N, Zeng H B, Tian Y. Recent advances in gecko adhesion and friction mechanisms and development of gecko-inspired dry adhesive surfaces. Friction 1(2): 114-129 (2013)

[6] Autumn K, Sitti M, Liang Y A, Peattie A M, Hansen W R, Sponberg S, Kenny T W, Fearing R, Israelachvili J N, Full $\mathrm{R}$ J. Evidence for van der Waals adhesion in gecko setae. Proc Natl Acad Sci USA 99(19): 12252-12256 (2002)

[7] Autumn K, Liang Y A, Hsieh S T, Zesch W, Chan W P, Kenny T W, Fearing R, Full R J. Adhesive force of a single 
gecko foot-hair. Nature 405(6787): 681-685 (2000)

[8] Stork N E. Experimental analysis of adhesion of Chrysolina polita (Chrysomelidae: Coleoptera) on a variety of surfaces. J Exp Biol 88(1): 91-107 (1980)

[9] Huber G, Mantz H, Spolenak R, Mecke K, Jacobs K, Gorb $\mathrm{S} N$, Arzt E. Evidence for capillarity contributions to gecko adhesion from single spatula nanomechanical measurements. Proc Natl Acad Sci USA 102(45): 16293-16296 (2005)

[10] Prevenslik T. Electrostatic Gecko Mechanism. Tribol Ind 31(1-2): 61-66 (2009)

[11] Izadi H, Penlidis A. Polymeric bio-inspired dry adhesives: van der Waals or electrostatic interactions? Macromol React Eng 7(11): 588-608 (2013)

[12] Harper W R. Contact and Frictional Electrification. Morgan Hill (USA): Laplacian Press, 1998.

[13] Lowell J, Rose-Innes A C. Contact electrification. Adv Phys 29(6): 947-1023 (1980)

[14] Izadi H, Stewart K M E, Penlidis A. Role of contact electrification and electrostatic interactions in gecko adhesion. J R Soc Interface 11(98): 20140371 (2014)

[15] Full R J, Tu M S. Mechanics of six-legged runners. J Exp Biol 148(1): 129-146 (1990)

[16] Dai Z D, Wang Z Y, Ji A H. Dynamics of gecko locomotion: a force-measuring array to measure 3D reaction forces. J Exp Biol 214(5): 703-708 (2011)

[17] Reedyk C W, Perlman M M. The measurement of surface charge. J Electrochem Soc 115(1): 49-51 (1968)

[18] Bassen H I, Smith G S. Electric field probes-A review. Ieee Tranc Antenn Propag 31(5): 710-718 (1983)

[19] Greason W D. Investigation of a test methodology for triboelectrification. J Electrostat 49(3-4): 245-256 (2000)

[20] Seyam A M F, Cai Y Y, Oxenham W. Devices for measuring electrostatic generation and dissipation on the surfaces of polymeric materials. J Text Instit 100(4): 338-349 (2009)

[21] Zhang Y Y, Shao T M. A method of charge measurement for contact electrification. $J$ Electrostat 71(4): 712-716 (2013)

[22] Chiou Y C, Chang Y P, Lee R T. Tribo-electrification mechanism for self-mated metals in dry severe wear process: Part I. pure hard metals. Wear 254(7-8): 606-615 (2003)

[23] Budakian R, Putterman S J. Correlation between charge transfer and stick-slip friction at a metal-insulator interface. Phys Rev Lett 85(5): 1000-1003 (2000)
[24] Gady B, Reifenberger R, Rimai D S, DeMejo L P. Contact electrification and the interaction force between a micrometer-size polystyrene sphere and a graphite surface. Langmuir 13(9): 2533-2537 (1997)

[25] Liu S H, Wei G H, Liu Z C. Electrostatic Theory and Electrostatic Protection. Beijing (China): Weapons Industry Press, 1999.

[26] Wu Q, Ji A H, Wang Z Y, Dai Z D. Improvement and test for a force sensor's natural frequency. Chin. J. Sens. Actuat. 23(2): 235-238 (2010)

[27] Cheng D K. Field and Wave Electromagnetics. 2nd ed. New York: Addison-Wesley, 1989.

[28] ESDEMC Technology. ES111 digital static charge meter. User's Manual (2011)

[29] Huber G, Gorb S N, Hosoda N, Spolenak R, Arzt E. Influence of surface roughness on gecko adhesion. Acta Biomater 3(4): 607-610 (2007)

[30] Maladen R D, Ding Y, Li C, Goldman D I. Undulatory swimming in sand: subsurface locomotion of the sandfish lizard. Science 325(5938): 314-318 (2009)

[31] Autumn K, Hsieh S T, Dudek D M, Chen J, Chitaphan C, Full R J. Dynamics of geckos running vertically. J Exp Biol 209(2): 260-272 (2006)

[32] Wang Z Y, Wang J T, Ji A H, Zhang Y Y, Dai Z D. Behavior and dynamics of gecko's locomotion: The effects of moving directions on a vertical surface. Chin Sci Bull 56(6): 573-583 (2011)

[33] Lacks D J, Sankaran R M. Contact electrification of insulating materials. J Phys D Appl Phys 44(45): 453001 (2011)

[34] Alibardi L. Cell biology of adhesive setae in gecko lizards. Zoology 112(6): 403-424 (2009)

[35] Apodaca M M, Wesson P J, Bishop K J M, Ratner M A, Grzybowski B A. Contact electrification between identical materials. Angew Chem 122(5): 958-961 (2010)

[36] Lowell J, Truscott W S. Triboelectrification of identical insulators. II. Theory and further experiments. $J$ Phys $D$ Appl Phys 19(7): 1281-1298 (1986)

[37] Eason E V, Hawkes E W, Windheim M, Christensen D L, Libby T, Cutkosky M R. Stress distribution and contact area measurements of a gecko toe using a high-resolution tactile sensor. Bioinspir Biomim 10(1): 16013 (2015)

[38] Wang S H, Lin L, Xie Y N, Jing Q S, Niu S M, Wang Z L. Sliding-triboelectric nanogenerators based on in-plane chargeseparation mechanism. Nano Lett 13(5): 2226-2233 (2013) 


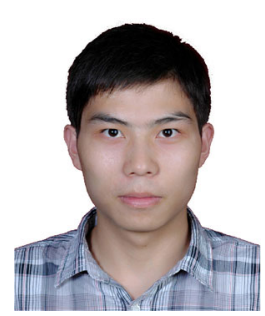

Yi SONG. He received his bachelor and master degrees in engineering mechanics from Nanjing University of Aeronautics and Astronautics (NUAA), Nanjing, China, in 2012

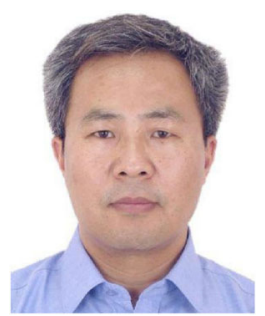

Zhendong DAI. He is a professor, a supervisor of $\mathrm{PhD}$ students, and the director of Institute of Bioinspired Surface and Engineering in Nanjing University of Aeronautics and Astronautics (NUAA), Nanjing, China. He obtained his Ph.D. degree and 2015, respectively. He became a Ph.D. student in mechanical design and theory at NUAA in 2015 and his research interests include bio-tribology, biomechanics, and bio-inspired adhesion.

in 1999 from College of Mechanical and Electrical Engineering, NUAA. After completing his postdoctoral research in 2001 in Max Planck Institute for Developmental Biology, he joined NUAA as a professor. His research areas include bionics, light material, control of bionics, bio-robots, and biological robots. 